\title{
Effects of mean strain and tensile pre-strain on torsional fatigue behaviours of duplex stainless steel SAF2205
}

\author{
Bingbing $\mathrm{Li}^{1}$, Dunji $\mathrm{Yu}^{1}$, and $X u$ Chen ${ }^{1, *}$ \\ ${ }^{1}$ School of Chemical Engineering and Technology, Tianjin University, Tianjin 300350, China
}

\begin{abstract}
Effects of mean strain and tensile pre-strain were investigated on the torsional fatigue behaviours of duplex stainless steel SAF2205. Two equivalent strain amplitudes $(0.5 \%, 0.7 \%)$, three strain ratios $(-1,-$ $0.5,-0.25)$ and $5 \%$ tensile pre-strain were chosen. Results indicated that the mean strain had no distinct influences on the torsional fatigue behaviours in terms of cyclic stress reponse and fatigue life while tensile pre-straining made a significant increase in cyclic stress response which was mainly attributed to the cross hardening derived from the loading sequence of monotonic tension preceding to cyclic torsion and led to a reduction in fatigue life. The failure mechanisms were revealed by scanning electron microscope characterized by microcracks initiation at the extrusions in ferrite and phase boundary inhibited further propagation. Additionally, the fractography of all fatigued specimens revealed a quasi-cleavage brittle mode with features of distinct tearing ridges and cleavage facets.
\end{abstract}

\section{Introduction}

Duplex stainless steels (DSS) beneficially combining the mechanical properties of both ferritic and austenitic phases in terms of strength, ductility and corrosion resistance are increasingly employed in highly aggressive environments such as nuclear power plants, automobile, chemical and petrochemical industries as a potential alternative to austenitic stainless steels (ASS) [1-6]. The structural components usually suffer alternative loadings resulting from the variable temperature fields and corresponding high stress fields. Hence, all the parameters which can influence the cyclic stress response and microstructural evolution should be evaluated comprehensively.

Investigations about the low cycle fatigue behaviours of DSS have been published mainly focused on the cyclic stress response, mechanisms of cracks nucleation and fatigue life [7-9,10-12]. PK Chiu et al. [7, 8] founded that a higher strain amplitude and tensile mean strain would promote the occurrence of strain-induced martensite (SIM) formation which could result in secondary hardening (SD). Additionally, V Aubin et al. [9] explored the fatigue properties for DSS X2CrNiMoN25-07 under complex biaxial loadings. The results showed that this material was not susceptible to the loading path as ASS did and presented a lower extrahardening on cyclic stress response, which should be attributed to the performance of each single phase under non-proportional fatigue where the austenite exhibited a strong over-hardening and contrarily little for the ferrite. However, information about the DSS LCF behaviours are too limited. The microcracks usually initiated along the extrusions in ferrite $[10,11]$ and ferrite grain boundaries [12]. Moreover, the phase boundaries usually worked as an effective barrier for microcracks to further propagate.

Pre-straining (cold work) recognized as an important fabrication processing variable has been universally employed in engineering construction during the fabrication of vessels, tanks, piping, heat exchangers, etc. Substantial references have been published focusing on the mechanisms of cold work/pre-straining on the LCF behaviours of ASS, aluminium and magnesium alloy [13-17], among which CL Zhou et al. [18, 19] investigate the effects of prior tensile plastic deformation on the torsional fatigue properties of a structural steel. As for the DSS, however, the researchers are mainly concerned with the effects of pre-straining/cold-work on the basic mechanical properties, microstructural evolution during monotonic loadings [20-24]. Much more efforts should be made to explore how it works on the subsequent LCF behaviours and reveals the corresponding mechanisms.

The present work aims at characterizing the torsional fatigue behaviours of DSS SAF2205 under various mean strain. In addition, much attention has been paid to the effects of monotonically tensile pre-straining on the subsequent torsional fatigue behaviours in order to get a better knowledge of the LCF behaviours of DSS sufficiently under complex loading conditions and supplement experimental data.

\section{Experimental procedure}

The material studied in the current research was DSS SAF 2205 containing a volume fraction $(\%)$ of ferrite $(\alpha)$ and austenite $(\gamma)$ of 53:47 which was manufactured in the 
process of cold-rolled and a thermal treatment at $1050^{\circ} \mathrm{C}$ followed by a rapid water quench. The chemical composition of alloying elements in weight percent (\%) is: $\mathrm{C} 0.019, \mathrm{~N} 0.192, \mathrm{Ni} 4.74, \mathrm{Cr} 22.3$, Mn 1.64, Mo 3.13, Si 0.41, S 0.001, P 0.018, Fe Bal. For the metallography microstructures observation of both phases, the material of as-received were ground and polished with sequentially finer grits down to $0.25 \mu \mathrm{m}$ diamond paste and then electrolytically etched in a solution of $10 \%$ oxalic acid at $20 \mathrm{~V}$ for $5 \mathrm{~s}$. It can be found that the austenitic islands were embedded in ferrite matrix with both phases distributed homogeneously in the transversal section meanwhile a highly elongated shape along the rolling direction was revealed (Fig.1). The surface and fractographic morphology of specimens after failure were observed using a HITACHI S-4800 scanning electron microscope (SEM).
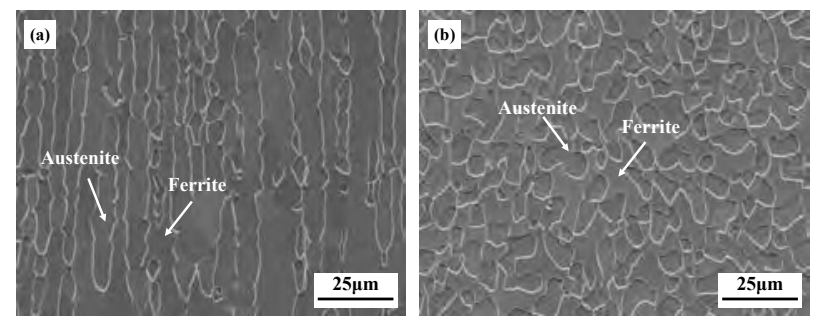

Fig. 1. Microstructure of DSS SAF2205 in longitudinal (a) and transversal (b) sections of as-received specimen.

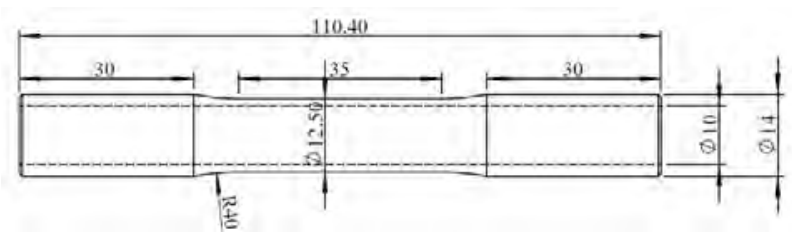

Fig. 2. Dimension of specimens used in current work.

Two equivalent strain amplitudes of $0.5 \%$ and $0.7 \%$ were chosen. Three strain ratios $(-1,-0.5$ and -0.25$)$ corresponding to various mean strain and $5 \%$ tensile prestraining were employed to investigate the effects of mean strain and monotonically tensile pre-straining on subsequent torsional fatigue behaviours. A servohydraulic MTS axial-torsional instrument and a tensiontorsion extensometer for measuring and controlling the shear strain were used. The thin wall-hollow tubular specimen was chosen in order to obtain the uniform distribution of shear stress over the transverse section and the dimension was schematically presented in Fig.2, as per ASTM E2207-08.

\section{Results and discussion}

\subsection{Effects of mean strain}

Table 1 and Fig. 3 exhibited the relevant parameters configuration and evolution of cyclic stress response of tests under various mean strain, respectively. Tests with strain range of $1.0 \%$ presented a consistent initial hardening followed by a continuous softening and finally a drastic reduction of stress amplitude resulting from the formation of macrocracks whatever the strain ratio was. Namely, the mean strain had almost no influence on the torsional fatigue behaviours in terms of cyclic stress response as shown in Fig. 3(a). From the perspective of fatigue life, the same conclusion seemed to be true yet in which specimens with different strain ratios gave a nearly equal life except tests with strain ratio of -0.25 which given a relatively longer life owing to the lower stress amplitude in the course of cycling. As for tests with strain range of $1.4 \%$, an extra stage of cyclic stabilization after a prolonged period of softening (Fig. 3(b)) developed comparing to that of test with strain range of $1.0 \%$. Fig.4(a) and (b) showed the two groups of hysteresis loops of tests with strain range $1.0 \%$ and $1.4 \%$ at half life time, respectively. It could be found that the hysteresis loops corresponding to different mean strain almost coincided at constant strain range, which indicated that the mean strain didn't bring about extra influence of beneficial or detrimental to the torsional fatigue behaviours viewed from the plastic strain energy point. Besides, the value of mean stress caused by asymmetrical strain was small relative to the cyclic stress amplitude and relaxed quickly with cycling (Fig. 5). Furthermore, the phenomenon of secondary hardening before final failure developed at tests with strain ratio of -0.25 . The stage of secondary hardening, even though the degree of which was quite slight lasted more than half of the fatigue life. The local amplification at the lower-left of Fig. 3(b) was made for clearer observation.

Table 1. Test parameters with mean strain.

\begin{tabular}{ccccc}
\hline $\mathbf{R}$ & $\Delta \boldsymbol{\gamma} \mathbf{( \% )}$ & $\gamma_{\min } / \gamma_{\max }$ & $\gamma_{\text {mean }}$ & Life \\
\hline \multirow{2}{*}{$\mathbf{1}$} & 1.0 & $-0.50 / 0.50$ & 0 & 7126 \\
& 1.4 & $-0.70 / 0.70$ & 0 & 3304 \\
$\mathbf{- 0 . 5}$ & 1.0 & $-0.33 / 0.67$ & 0.17 & 6260 \\
& 1.4 & $-0.47 / 0.93$ & 0.23 & 3174 \\
$\mathbf{- 0 . 2 5}$ & 1.0 & $-0.20 / 0.80$ & 0.3 & 10366 \\
& 1.4 & $-0.28 / 1.12$ & 0.42 & 5438 \\
\hline
\end{tabular}

The fatigue behaviours resulting from cyclic plasticity is linked to the formation, movement and annihilation of dislocations. Therefore, the evolution of cyclic stress response is strongly dependent on the dislocation configuration and evolution of microstructures. GVP Reddy et al. [25] determined that the cyclic hardening was the result of the individual or combined of 1) dislocation reproducible and mutual interactions, 2) dislocation-precipitations interactions acting as local obstacles on the glide plane, 3) dislocation-mobile dislocations, namely DSA. [26] measured the Vickers hardness and fraction of martensite of specimens at cycles corresponding to the initial hardening region and found a large increase in hardness with almost no increase in the amount of martensite, which implied the initial hardening should be attributed to the increase in dislocation density during fatigue. Additionally, the progressive build-up of dislocation pile-ups during cycling which will induce an increase in the resistance of grain boundaries or obstacles to the further pile-ups might be another source of initial hardening in addition to the increase in 
dislocation density. With regard to the softening which is quitely sensitive to the microstructural changes mainly to the rearrangement of dislocation configuration, factors which can influence the movement and evolution of dislocation, such as chemical composition, temperature, strain amplitude and strain rate will make a distinct contribution to the cyclic softening. The addition of $\mathrm{N}$ into the matrix will promote the trend of slip planarity and slip reversibility. The nitrogen addition to $316 \mathrm{LN}$ stainless steel has been reported to induce cyclic softening at room temperature [27, 28]. Similarly, the same effect was also observed in DSS Fe-Cr-Mn-N. The higher strain amplitude will bring out a larger plastic component which can result in a high density of mobile dislocations to make further efforts to the softening. In addition, the stabilization was closely related to the formation of well-defined low energy configuration (such as dislocation cell).
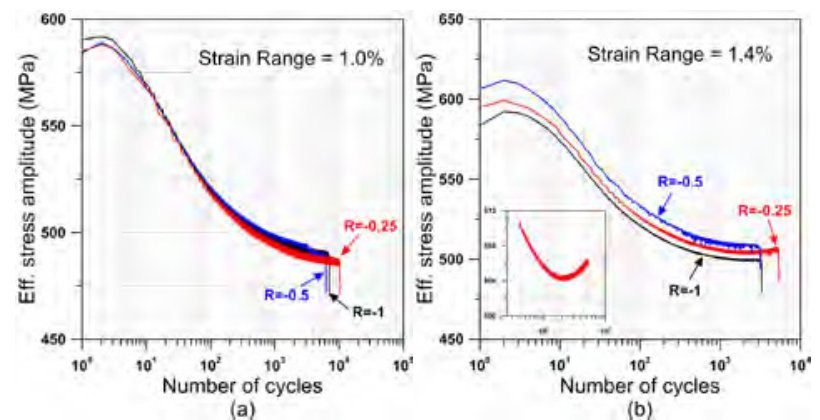

Fig. 3. Cyclic stress response under different strain ranges and strain ratios.

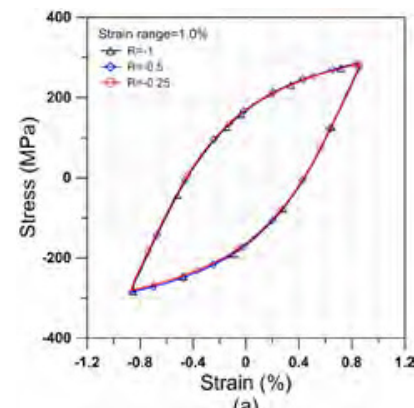

(a)

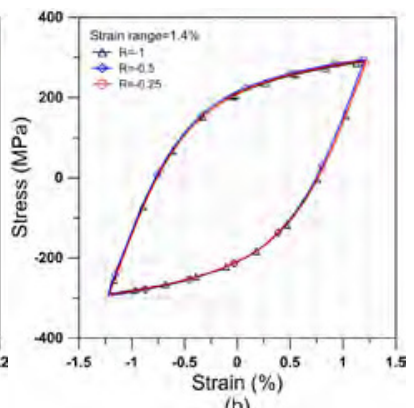

(b)
Fig. 4. Hysteresis loops at half life time.

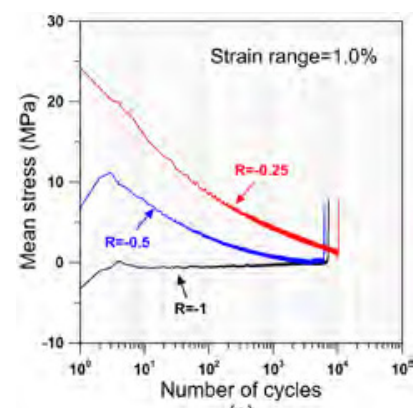

(a)

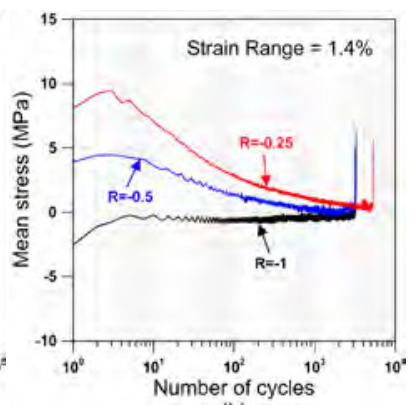

(b)
Fig. 5. Mean stress versus number of cycles at various loading conditions.

Plenty of investigations have attributed the occurrence of secondary hardening to the strain induced martensite (SIM) transformation pertaining to stainless steel especially ASS or DSS at room temperature [29, 30]. Despite the low stacking fault energy (SFE) which can prompt the SIM formation of DSS, the steel was found to be more stable than other stainless-steel grades, such as AISI 304 or 316 and its variants [23] which was agreement with the results in the present work in which the degree of secondary hardening was quite slight. There were usually two types of martensite formed during the course of SIM, hexagonal $\varepsilon$-martensite phase and $\mathrm{BCC}-\alpha$ '-martensite occurred directly from austenite or via the hexagonal $\varepsilon$-martensite phase [23,31]. Additionally, P.K. Chiu [7] proposed that the presence of strain-induced intersected $\varepsilon$-martensite bands is more beneficial and preferential than the $\alpha^{\prime}$-martensite to the fatigue properties of SAF2205. In conclusion, there was no distinct influence of mean strain on the torsional fatigue behaviours at shear strain range of $1.0 \%$ and $1.4 \%$ in the current work.

\subsection{Effects of tensile pre-straining}

The consistent trend of continuous cyclic softening until final failure characterized was detected irrespective of strain amplitudes illustrated in Fig. 6. Additionally, the higher the shear strain amplitude, the higher softening rate obtained. Reference [32] reported that the fatigue behaviours showed strong propensity to the loading history for materials with a low SFE. The cyclic softening which can reduce dislocation density and promote microstructural rearrangement leading to finally the formation of low energy dislocation structures was a dynamic recovery process induced by cyclic loading [13, $33,34]$. It has been extensively investigated that the material subjected to a prior plastic deformation will present continuously cyclic softening in the subsequent pull-push fatigue tests $[14,31-33,35,36]$. As mentioned in section 3.1, the high dislocation density was responsible to the initial hardening, thus, the prestraining stage producing plenty of dislocations could be regarded as an equivalent process to the initial cycling. What's more, the stored strain energy ascribed to the high tensile pre-deformation will work as the driving force to accelerate the rearrangement process, both of which worked together to cause the continuous softening during the whole life process. C. larid et.al [33] reported that the softening would occur if a specimen initially cycled at a high strain amplitude and subsequently cycled at a low strain amplitude, or if severe monotonic deformation was followed by fatigue. Results showed that the dislocation structures were strongly strain amplitude dependent in which loop patches at low strain amplitudes, mixed with dipolar wall structures at intermediate amplitudes and increasingly cellular at high strain amplitudes was revealed. In addition, the dislocation cell became increasingly equiaxed and small size with increase of strain amplitude. The initial cyclic hardening stage preceding to the softening can be found for 316 ASS in the annealed state owing to the thermal activation recovery process which can reduce the original dislocation density before the followed fatigue tests and provide enough capacity for new dislocation 
nucleation while a substantial cyclic softening was observed, as for the cold worked condition [36]. The strain-controlled fatigue behaviour of cold-drawn type 316 austenitic stainless steel underwent various levels of pre-straining has been investigated at strain amplitudes varying from $0.22 \%$ to $0.75 \%$ and at room temperature. The results showed that continuous cyclic softening occurred over the whole lifetime at strain amplitude higher than $0.35 \%$ in the $20 \%$ cold-drawn steel, and $0.31 \%$ in the $30 \%$ cold-drawn steel. The speed for dislocation formation was still higher than annihilation at small strain amplitude leading to the occurrence of initial hardening, which implied that there was a threshold strain required for cyclic softening during fatigue. Only the applied strain amplitude was high enough, can the material obtain sufficient driving force for the rearrangement $[13,26]$.

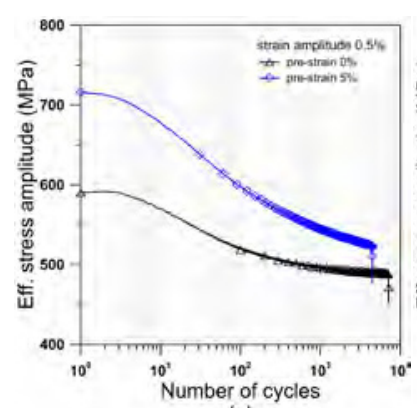

(a)

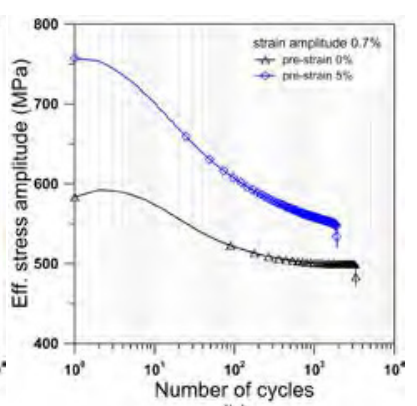

(b)
Fig. 6. Cyclic stress response under different strain amplitudes and pre-straining levels.
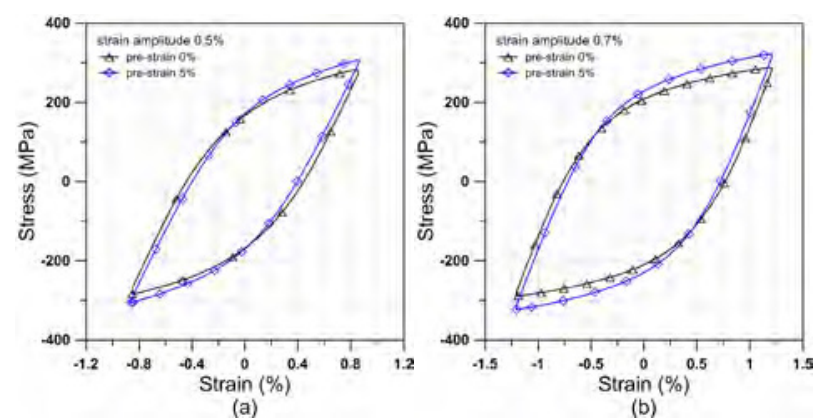

Fig. 7. Hysteresis loops at half life time.

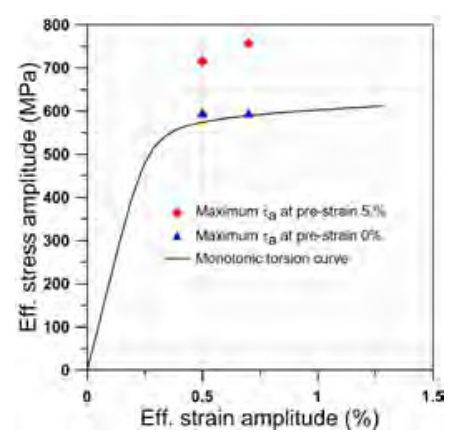

Fig. 8. Schematic illustration to significantly cyclic hardening for tests with pre-straining.

Fig. 6 depicted the relation of stress amplitude to number of cycles at strain amplitude $0.5 \%$ and $0.7 \%$ after $5 \%$ tensile prior plastic deformation where a significantly enhanced cyclic hardening can be observed.
The pre-straining could improve strength and decrease ductility which can be verified by Fig. 7 in which a gradual reduction in the plastic strain amplitude with the increase of pre-straining levels can be observed. Tensile pre-straining indicates loading the material to one certain value monotonically and then unloading, which has been recognized as an important processing variable for industrial application to improve the material strength $[13,14,26]$. Huang [37] gave the explanation for the considerable cyclic hardening from perspective of dislocation glide, deformation induced twinning and martensite transformation. The increase of dislocation density enhancing the interaction in a short-range order which further inhibited the dislocation movement and formation of twinning particularly for material with low SFE was the predominant mechanism at the early stage. The strain-induced martensite transformation began to control the deformation process after the amount of twinning reaching saturation. The loading sequence of tensile pre-straining followed by torsional fatigue which was quite similar to the non-proportional cross loading path which will introduce a considerable cross-hardening effect. However, the loading profile in the present work just equaled to only one cycle of cross loading path for the tensile loading applied in the pre-straining process was just monotonic. It can be observed that the maximum stress amplitude of tests without pre-straining at different strain amplitudes were larger than that determined by monotonic curve at the identical strain amplitude which can be ascribed to the effect of cyclic hardening (Fig.8). Therefore, the difference between maximum stress amplitudes at tests without pre-straining and that at tests with pre-straining can be taken as simply the effect of cross-hardening. Moreover, the effect of cross-hardening degraded gradually with cycling which indicated that the effect of cross-hardening would make an influence to the stress response in the subsequent cycling which was consistent with the progressively decreasing difference between stress amplitudes. The effect of cross-hardening derived from non-proportional cross-shaped loading path has been published [38, 39]

\subsection{Microstructural analysis}

The failure model of all the tests in the present work irrespective of strain amplitudes, mean strain and prestraining can be consolidated into the shear-type, which was characterized by a primary macrocrack propagate along the maximum shear stress plane, namely parallel to the specimen axis (Fig. 9(a)). The Fig. 9(b) demonstrated the manner of cracks propagation of one representative fractography morphologies in detail. The outer surface usually became the preferential sites for cracks initiation and then propagating along radial direction toward inner surface by a manner of circulararc-shaped. The propagation toward the two ends of specimen began simultaneously after approaching a large depth, which finally induced the primary macrocracks. The well-defined tearing ridges at the lower left of Fig. 9(c) presented the typical features of brittle quasicleavage fracture. Fig. 10 compared the morphology of 
stable propagation regions of tests with $5 \%$ pre-straining and without pre-straining to investigated the effects of tensile prior deformation on the cracks behaviours. The well-defined cleavage facets could be found at tests without pre-straining. Further, the number of secondary cracks decreased meanwhile the size increased considerably attributing to the coalescences of small cracks owing to the pre-straining, which finally evolved into the macrocracks parallel to the specimen axis. In addition, the characteristics of brittle fracture mainly referring to the cleavage facets became less and less obvious.
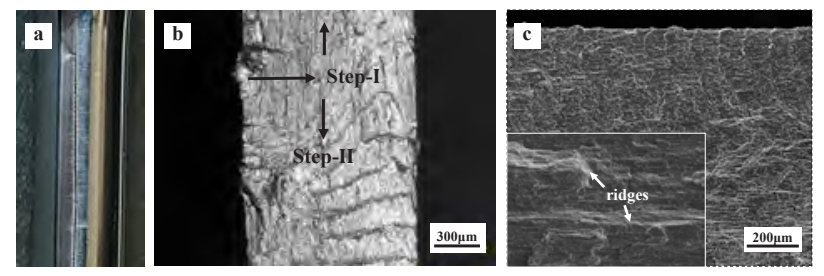

Fig. 9. (a)Morphology of primary cracks by camera, (b) Illustration of cracks propagation behaviours, (c)Different regions of fracture surface over radial direction
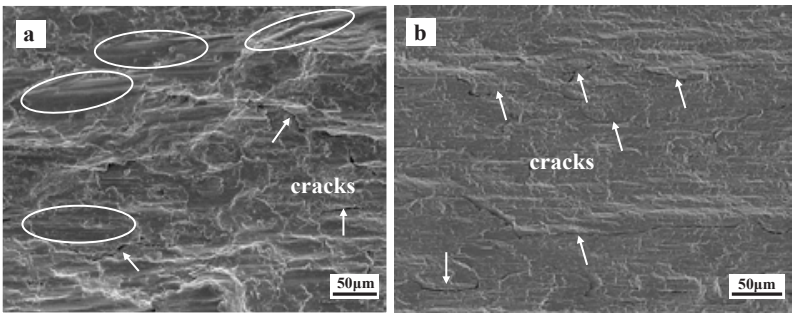

Fig. 10. Morphology of propagation regions on longitudinal surfaces at strain amplitude of $0.5 \%$ under loading conditions of (a)without pre-straining, (b)5.0 pre-straining.
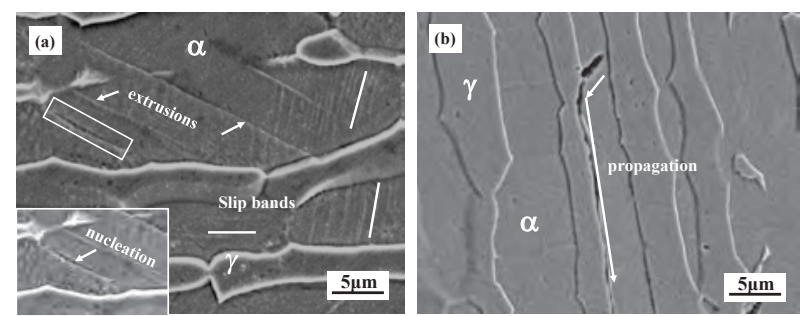

Fig. 11. Microcracks nucleation and propagation at extrusions in ferrite.

The majority of the plastic deformation should occur mainly in the ferritic phase which was consistent with the SEM observations of fatigued specimens as shown in Fig. 11(a). The substructure of intense slip marks formed in ferrite firstly and then microcracks nucleated along the extrusions. Besides, the flow of plasticity was nearly inhibited in the ferrite and the slip marks cannot pass through the phase boundary when the crystallographic Kurjumov-Sachs $(\mathrm{K}-\mathrm{S})$ relationships was not fulfilled. Thus, after nucleation the microcracks continued propagating along the phase boundary which was parallel to the specimen axis (Fig. 11(b)). Similar conclusions have also been found that the preferential sites for microcracks nucleation are the extrusions in ferritic phase and the phase boundary will hinder further propagation as an effective barrier [40].

\section{Conclusions}

A series of strain-controlled fatigue tests were carried out to investigate the effects of mean strain and prior tensile plastic deformation on torsional fatigue behaviours of DSS SAF2205 primarily in terms of cyclic stress response, fractographic morphology analysis and mechanisms of microcracks nucleation. After careful observation and discussion, conclusions can be drawn:

(1) A mixed model including initial cyclic hardening followed by a long period of softening and/or stabilization was observed in the current research. The slight secondary hardening developed at strain amplitude $0.7 \%$ jointed with a relative higher mean strain $(\mathrm{R}=-0.25)$ attributed to the occurrence of SIM transformation.

(2) The mean stress resulted from asymmetrical loading profile was small and relaxed quickly, which made little influence on the torsional fatigue behaviours in terms of cyclic stress response and fatigue life.

(3) Specimens subjected to the tensile pre-straining exhibited a continuous cyclic softening over the whole lifetime. Moreover, cross-hardening derived from the loading sequence of large tensile plastic deformation preceding to the torsional cyclic loading made a significantly extra cyclic hardening.

(4) A shear-type failure with features of a primary long crack propagating along the maximum shear stress plane was illustrated. More specifically, the microcracks nucleated at the outer surface and then propagated along the radial direction and further propagation along the axial direction. The fractography morphology of all fatigued specimens revealed a quasi-cleavage brittle mode characterized by distinct cleavage steps, tearing ridges and small cleavage facets.

\section{References}

1. R. Lillbacka, G. Chai, M. Ekh, P. Liu, E. Johnson, $\mathrm{K}$.

Runesson, Acta Mater. 55, 5359 (2007)

2. R. Strubbia, S. Hereñú, M.C. Marinelli, I. AlvarezArmas, Mater. Sci. Eng., A 65947 (2016)

3. S. Emami, T. Saeid, R.A. Khosroshahi, J Alloys Compd 739678 (2018)

4. H. Luo, X.G. Li, C.F. Dong, K. Xiao, Arabian J. Chem. 10 S90 (2017)

5. L. Jinlong, L. Tongxiang, W. Chen, D. Limin, Mater.

Sci. Eng., C 62558 (2016)

6. A.G. Terricabras, C.G. Schön, Mater. Sci. Eng., A 71720 (2018)

7. P.K. Chiu, K.L. Weng, S.H. Wang, J.R. Yang, Y.S. Huang, J. Fang, Mater. Sci. Eng., A 398349 (2005)

8. P.K. Chiu, S.H. Wang, J.R. Yang, K.L. Weng, J. Fang, 
Mater. Chem. Phys. 98103 (2006)

9. P.Q. Ve'ronique Aubin, S. Degallaix, Mater. Sci. Eng., A, 346208 (2003)

10. R. Strubbia, S. Hereñú, M.C. Marinelli, I. AlvarezArmas, Int. J. Fatigue 4190 (2012)

11. R. Strubbia, S. Hereñú, A. Giertler, I. AlvarezArmas,

U. Krupp, Int. J. Fatigue 6558 (2014)

12. M.-C. Marinelli, A. El Bartali, J.W. Signorelli, P. Evrard, V. Aubin, I. Alvarez-Armas, S. DegallaixMoreuil, Mater. Sci. Eng., A, 50981 (2009)

13. S.G.S. Raman, K.A. Padmanabhan, Int. J. Fatigue 18 71 (1996)

14. K.B.S. Rao, M. Valsan, R. Sandhya, S.L. Mannan,

P. Rodriguez, Metall. Trans. A 24913 (1993)

15. B. Wang, Z.J. Zhang, C.W. Shao, Q.Q. Duan, J.C. Pang, H.J. Yang, X.W. Li, Z.-F. Zhang, Metall.

Mater. Trans. A 463317 (2015)

16. K.S. Al-Rubaie, M.A. Del Grande, D.N. Travessa, K.R. Cardoso, Mater. Sci. Eng., A 464141 (2007)

17. T. Hama, H. Nagao, Y. Kuchinomachi, H. Takuda, Mater. Sci. Eng., A 59169 (2014)

18. C.L. Zhou, S.I. Nishida, N. Hattori, Key Eng. Mater. 353-358 94 (2007)

19. C.L. Zhou, S.I. Nishida, N. Hattori, Key Eng. Mater. 340-341 507 (2007)

20. J.L. KH Lo, CH Shek, D.J. Li, Mater. Sci. Eng., A s452-453 78 (2007)

21. S.S.M. Tavares, J.M. Pardal, M.R.D. Silva, C.A.S.D. Oliveira, Mater. Res. 17381 (2014)

22. M. Breda, K. Brunelli, F. Grazzi, A. Scherillo, I. Calliari, Metall. Mater. Trans. A 46577 (2014)

23. S.S.M. Tavares, M.R. da Silva, J.M. Pardal, H.F.G. Abreu, A.M. Gomes, J. Mater. Process. Technol.

180318 (2006)

24. S. Pramanik, S. Bera, S.K. Ghosh, Steel. Res. Int. 85 776 (2014)

25. G.V.P. Reddy, R. Sandhya, S. Sankaran, P. Parameswaran, K. Laha, Mater. Des. 88972 (2015)

26. Y.C. Jeon, C.S. Kim, H. Ki, S.I. Kwun, J.W. Byeon, Mater. Sci. Forum 580-582 597 (2008)

27. B.K. Choudhary, Metall. Mater. Trans. A 45302 (2013)

28. G.V.P. Reddy, R. Sandhya, S. Sankaran, M.D. Mathew, Mater. Trans. A 455044 (2014)

29. M. Okayasu, H. Fukui, H. Ohfuji, T. Shiraishi, J. Mater. Sci. 486157 (2013)

30. Z.S. XUE Zong-yu, WEI Xi-cheng, J. Iron. \& Steel Res. (Int.) 1751 (2010)

31. J. SINGH, J. Mater. Sci. 203157 (1985)

32. A. Belattar, L. Taleb, A. Hauet, S. Taheri, Mater.

Sci. Eng., A 536170 (2012)

33. Z.W. C Laird, B.T. Ma, H.F. Chai, Mater. Sci. Eng., A 113245 (1989)
34. C.L. HF Chai, Mater. Sci. Eng. 93159 (1987)

35. J.T. LF Coffin, Trans. Met. Soc. AIME 215794 (1959)

36. M.D. WJ Plumbridge, PJ Castle, Fatigue Fract. Eng. Mater. Struct. 3177 (2010)

37. K. HUANG Wenke, Acta Metall. Sinica (English Letters) 45275 (2009)

38. E.T.H. Okuchi, Trans. Japan. Soc. Mech. Eng. A 54 1588 (1988)

39. L. Taleb, A. Hauet, Int. J. Plast., 251359 (2009)

40. R. Strubbia, S.Hereñú, I.Alvarez-Armas, U.Krupp, Mater. Sci. Eng., A 615169 (2014) 